\title{
Temperature and thickness dependence of the timescale of crystallization of polymers under 1D confinement
}

\author{
Bao Wang, Allen Mathew and Simone Napolitano* \\ Laboratory of Polymer and Soft Matter Dynamics, Experimental Soft Matter and Thermal Physics (EST), \\ Université libre de Bruxelles (ULB), Boulevard du Triomphe, Brussels 1050, Belgium
}

Supporting Information for

1.1 Measurement of PLLA crystal growth rate

1.2 Determination of Stokes-Einstein coefficient

1.3 Thermodynamic properties of PLLA $\alpha$ ' crystal

1.4 Thickness and temperature dependence of the segmental relaxation time 


\subsection{Measurement of PLLA crystal growth rate}

PLLA films with thickness around $10 \mu \mathrm{m}$ were first heated to $205{ }^{\circ} \mathrm{C}(3 \mathrm{~K}$ above the equilibrium melting point) and annealed for $3 \mathrm{~min}$ to relax mechanical stresses and erase thermal history. Then the films were cooled down to $135^{\circ} \mathrm{C}$ and held for $15 \mathrm{~min}$ to create sporadic PLLA spherulitic seeds. After that, the samples were quenched to the temperature of the crystal growth measurement, which was performed under polarized optical microscopy. As an example, the morphology of spherulite growth process at $85^{\circ} \mathrm{C}$ is shown in Figure S1. For each image, the radius of the spherulites was measured for at least three times along different directions. The growth rate $G$ was obtained via linear regression of the relative growth of the spherulite radius versus time, owing to the relation $R(t)=R_{0}+$ $G t$, where $R_{0}$ is the radius of the spherulite at time $t=0$.
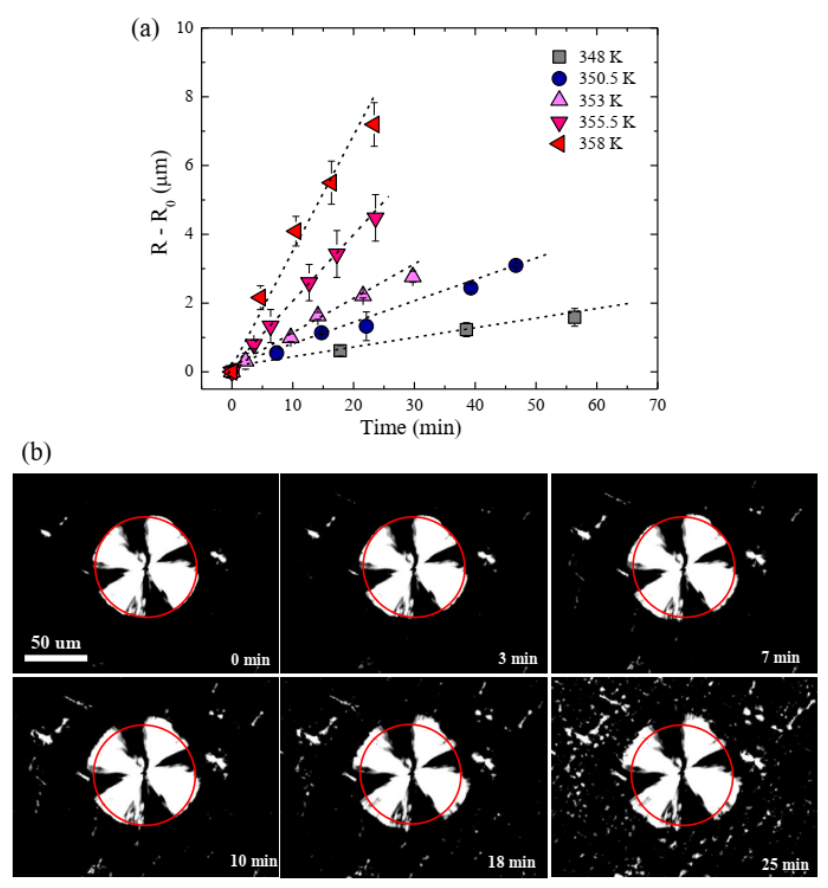

Figure S1. (a) Radius of PLLA spherulite with annealing time at different temperatures, $R$ is the radius of spherulite with time and $R_{0}$ is the initial radius; (b) Morphology of the PLLA spherulite during annealing at $85{ }^{\circ} \mathrm{C}$. 


\subsection{Determination of Stokes-Einstein coefficient}

The obtained PLLA crystal growth rates were plotted as a function of segmental relaxation time in a log-log plot, see Figure S2. According to the Stokes-Einstein equation, the growth rate of PLLA crystal, $G$, scales with the segmental relaxation time, $\tau_{\alpha}$, as $G \sim$ $\left(\tau_{\alpha} / T\right)^{-\xi} . \xi$ is the Stokes-Einstein coefficient, that usually shows value around $0.5-1.0$ in the proximity of $T_{g}{ }^{1}$ Since the temperature range used is narrow $(\Delta T=10 \mathrm{~K})$, the term $1 / T$ is almost constant $(\Delta T / T<<1)$ and can be neglected. Therefore, the value of $\xi$ can be obtained as the slope of the linear regression of the logarithm of the growth rate vs the logarithm of the segmental time, see Figure S2. The experimental values of the segmental relaxation are given in Figure S4.

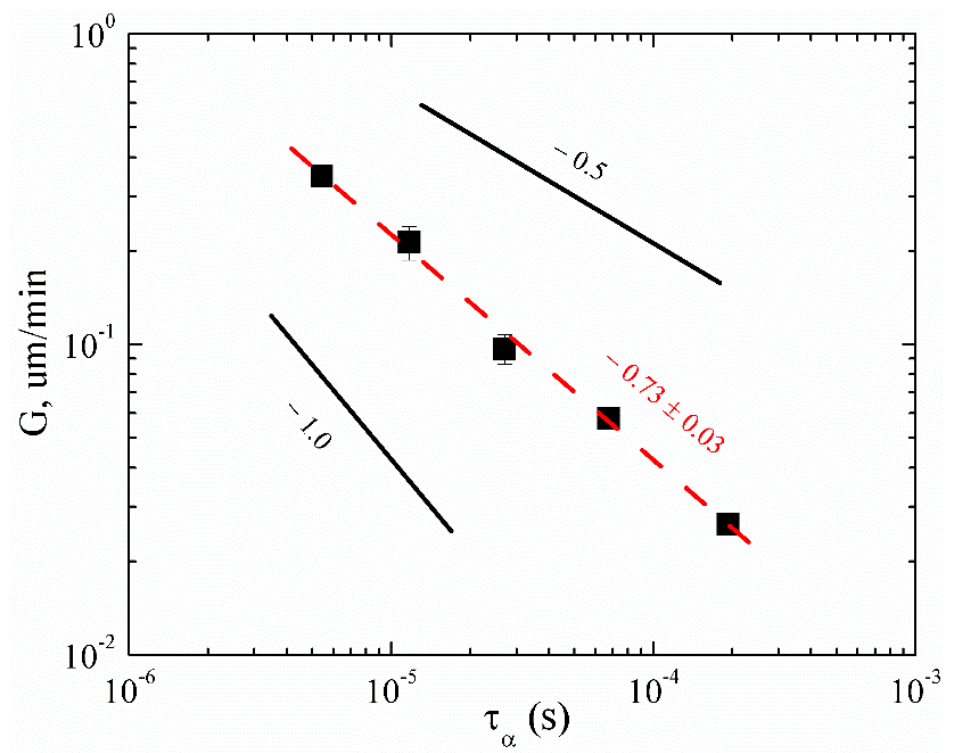

Figure S2. PLLA crystal growth rates as a function of segmental relaxation time. 


\subsection{Thermodynamic properties of PLLA $\alpha^{\prime}$ crystal}

According to the classical nucleation theory, the nucleation rate I is given by:

$$
\operatorname{Ln} I=\operatorname{Ln} I_{0}-\frac{\Delta E^{*}}{R\left(T_{c}-T_{\infty}\right)}-\frac{32 \sigma^{2} \sigma_{e} T_{m}^{0^{2}}}{k_{B} T_{c}\left(\Delta T \Delta h_{f}\right)^{2}}
$$

$I_{0}$ is a temperature independent nucleation constant, $R$ is the gas constant, $k_{B}$ is the Boltzmann constant and $\Delta E^{*}$ is the activation energy responsible for diffusion of crystallizable elements cross the phase boundary. $\sigma$ and $\sigma_{e}$ are the lateral- and end-surface free energy of crystal-melt interface, $T_{m}^{0}$ is the equilibrium melting temperature of PLLA crystal, $\Delta T=T_{m}^{0}-T_{c}$ is the undercooling, $\Delta h_{f}$ is the enthalpy of fusion per unit volume of PLLA crystal. Values of $\Delta E^{*}, T_{\infty}$ and $T_{m}^{0}$ for PLLA are taken from the literature, as $1.5 \mathrm{kcal}$ $\mathrm{mol}^{-1}, 300 \mathrm{~K}$, and $475 \mathrm{~K}$, respectively. ${ }^{2,3}$

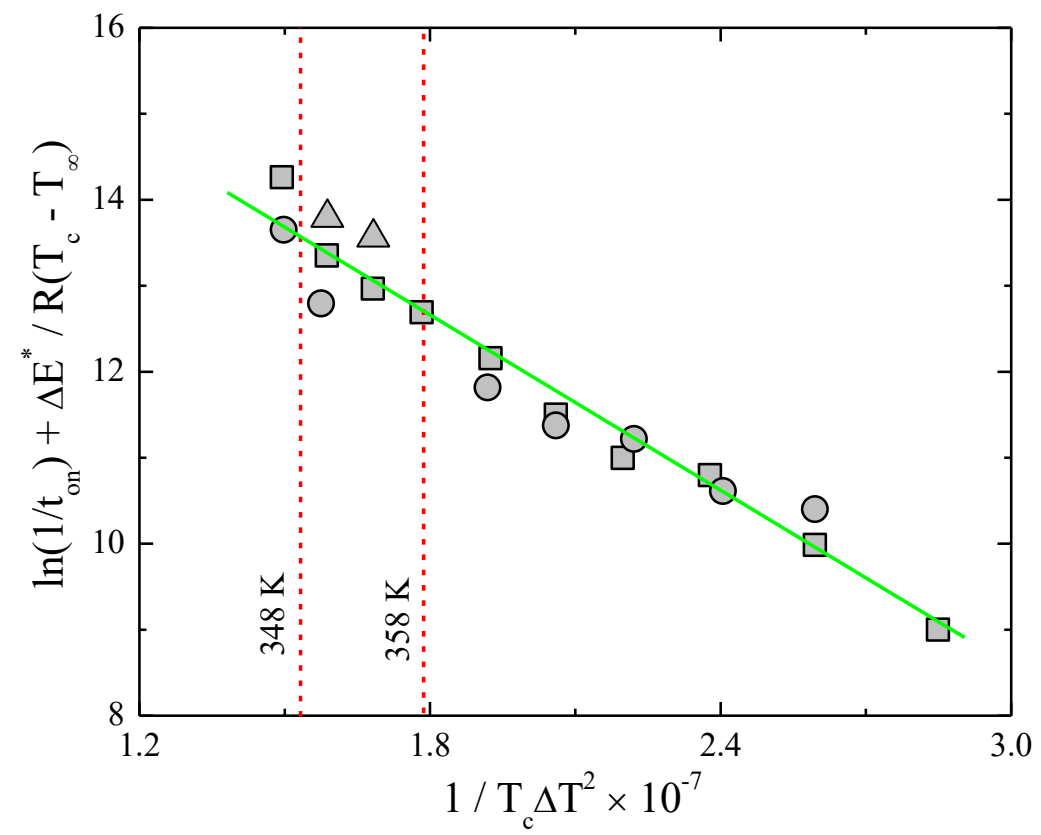

Figure S3. Onset time of PLLA crystal nucleation at different temperatures. The crystallization temperatures used in this work is between the red dashed lines. The data points with different shapes represent tests performed on different samples. 
Based on these quantities, we extrapolated the value of $32 \sigma^{2} \sigma_{e} T_{m}^{0^{2}} / k_{B} \Delta h_{f}^{2}$, necessary to obtain the energy barrier for nucleation $\Delta U^{*}$, from the data of Androsch et al, ${ }^{4}$ see Figure $\mathrm{S} 3$, who measured via flash calorimetry the onset time of nucleation, $t_{o n} \equiv I^{-1}$, in the temperature range of our experiments. By plotting $\ln \left(1 / t_{o n}\right)+\Delta E^{*} / R\left(T_{c}-T_{\infty}\right)$ vs $1 / T_{c} \Delta T^{2}$, a linear relationship with slope, $32 \sigma^{2} \sigma_{e} T_{m}^{0^{2}} / k_{B} \Delta h_{f}^{2}$ is finally obtained to be $(5.54 \pm 0.61) \times 10^{7}$ $\mathrm{K}^{3}$.

\subsection{Segmental relaxation time with film thickness at different temperatures}

The segmental relaxation time, $\tau_{\alpha}$, of amorphous PLLA at $343 \mathrm{~K}$ is plotted in Figure S4(a) as a function of the sample thickness. $\tau_{\alpha}$ is constant, within the experimental incertitude, until around $30 \mathrm{~nm}$.
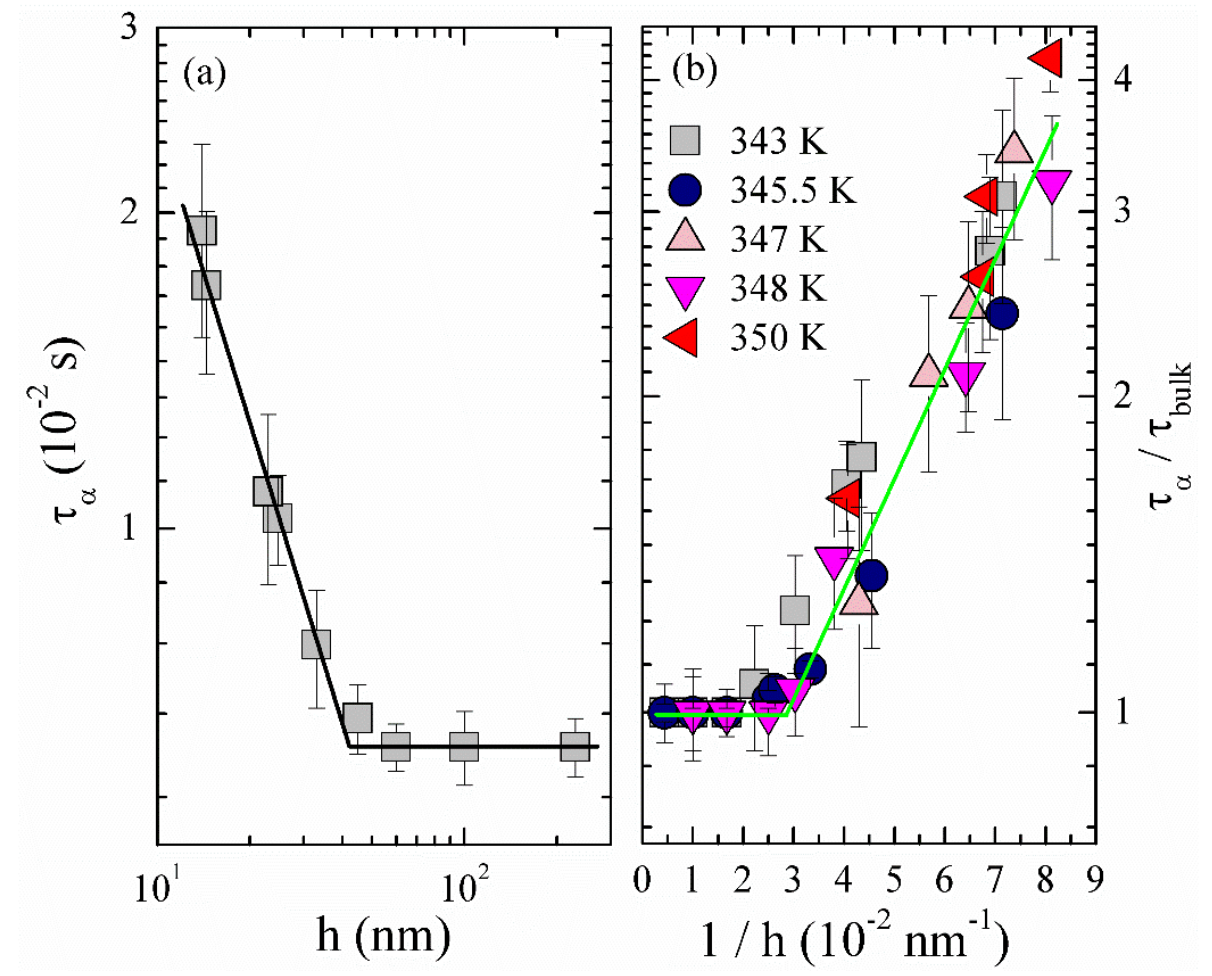

Figure S4. (A) PLLA segmental relaxation time with film thicknesses at $343 \mathrm{~K}$; (b) Onset time of PLLA crystal nucleation at different temperatures. The crystallization temperatures used in this work is between the red dashed lines. The data points with different shapes represent reptations with different samples. 
Below this threshold the segmental time dramatically increases upon confinement. Meanwhile, the ratio between the film value and the bulk value, $\tau_{\alpha} / \tau_{b u l k}$, shows the same trend at different temperatures. The thickness dependence of $\tau_{\alpha}$ can be described by:

$$
\ln \left(\frac{\tau_{\alpha}(h, T)}{\tau_{\text {bulk }}(T)}\right)=\left\{\begin{array}{cc}
0 & h \geq 30 \mathrm{~nm} \\
\frac{k}{h}-b & h<30 \mathrm{~nm}
\end{array}\right.
$$

where $\mathrm{k}$ and $\mathrm{b}$ are positive fitting constants, respectively equal to to $19.1 \pm 1.5 \mathrm{~nm}$ and 0.57 \pm 0.11 for the temperature range used in this work. The expression of $\tau_{\alpha}(h, T)$ was used for fitting and prediction Figure 1.

\section{References}

1. Hall, D. B.; Dhinojwala, A.; Torkelson, J. M. Translation-rotation paradox for diffusion in glassforming polymers: the role of the temperature dependence of the relaxation time distribution. Physical review letters 1997, 79 (1), 103.

2. Saeidlou, S.; Huneault, M. A.; Li, H.; Park, C. B. Poly (lactic acid) crystallization. Progress in Polymer Science 2012, 37 (12), 1657-1677.

3. Wang, B.; Wen, T.; Zhang, X.; Tercjak, A.; Dong, X.; Müller, A. J.; Wang, D.; Cavallo, D. Nucleation of Poly (lactide) on the Surface of Different Fibers. Macromolecules 2019, 52 (16), 62746284.

4. Androsch, R.; Di Lorenzo, M. L. Crystal nucleation in glassy poly (1-lactic acid). Macromolecules 2013, 46 (15), 6048-6056. 